\title{
Machine Vision-based Segmentation and Classification Method for Intelligent Roller Surface Monitoring
}

\author{
Depeng Shi, Jiehan Zhou, Jirui Xu, Jun Yang, Xuekun Li, Zeming Zhao, Junchuang Chen, Yiming \\ Rong
}

\begin{abstract}
The surface quality of steel rollers is a key factor determining the quality of final products such as metal sheets and foils in the rolling industry. It is important to examine the surface quality of rollers since rollers with optical defects will always duplicate the defects onto the metal sheet or foil during rolling. The typical optical defects of rollers after finish grinding include speckles, chatter marks, swirl marks and combination of all of the above. They can hardly be modeled or shaped by the approach of micro topography or SEM (scanning electrical microscope). In this paper, an on-site machine vision system is firstly applied for stable inspection for the optical defects on roller surfaces. Then, an improved optical defect segmentation algorithm is developed based on the active contour model and the images including chatter marks and swirl marks. The normal surface state is classified by the combination of methods of Gabor filters, KPCA method and ELM neural networks. Finally, experiment are carried out to verify the efficiency of the improved segmentation method and the recognition rate of the combined classification algorithm.
\end{abstract}

Keywords: optical defect detection, machine vision, big data, roller surface monitoring.

\section{INTRODUCTION}

In the field of extraordinary precision rolling, the surface quality of steel roller has significant influence on the surface integrity of products, which has been widely used in the military industry such as battery plates, military wire wrap foil. Since rollers' surface finish is always reduced gradually in use and the surface defect will reflect on the work piece surface, which always causes low quality products. Therefore, surface inspection is a critical step in the field of not only metal sheet or foil but also roller.

Most of the defects are too small to be visible for naked eye. However, current roller surface inspection still mainly relies on naked eye, which has low consistency, time consumption and high error rate. Therefore, there is a growing need for a real-time on-site roller surface-monitoring system.

*Resrach supported by Tsinghua University Initiative Scientific Research Program Tsinghua-RWTH Aachen Collaborative Innovation and Southern University of Science and Technology.

Depeng Shi is a postgraduate studying in the Department of MEE of Southern University of Science and Technology. China (e-mail: 11649096@mail.sustc.edu.cn).

Jiehan Zhou is a faculty of Information Technology and Electrical Engineering in Oulu University. Finland (e-mail: jiehan.zhou@oulu.fi).

Jirui $\mathrm{Xu}$ is a postgraduate studying in the Department of Mechanical Engineering at Tsinghua University, China (e-mail: xjr15@mails.tsinghua.edu.cn)
Machine vision is regarded as a suitable technology for stable and precise surface inspection. However, it is difficult to capture roller surface image due to large and high-gloss cylindrical roller surface and fine visual defects. Therefore, it requires an effective algorithm for defect identification, segmentation and classification. The existing classification algorithms are Gabor filters, Kernel Principal Component analysis (KPCA) and extreme learning machine (ELM), which can achieve a relatively high recognition rates.

For image segmentation, Kass et al. first proposed an active contour model (ACM) in 1988, also called Snake[1]. It relies on curve evaluation to detect interested objects based on the gradient of object edges. Malladi et al. proposed geometric active contours, taking advantages of level set method, which can effectively change curve shape in topology [2]. Shi et al. proposed a fast method without the need of solving the partial differential equations [3]. Caselles et al. proposed a geodesic active contour model [4]. Above mentioned algorithms are all edge-based models, which often use an edge indicator to attract the curve towards the target boundaries. They are efficient for series of image segmentation. However, they have difficulty detecting edges without obvious gradients, like roller surface's fuzzy and blur boundary.

Chan et al. proposed another well-known region-based model, called active contour model without edges (Chan-Vese model) [5]. This model is based on image region instead of edges, using global image gray intensity rather than edge gradient. They further proposed Piecewise Constant (PC) model and used the level set function to solve multiphase segmentation [6]. Both Chan-Vese and PC models cannot work effectively when illumination is inhomogeneous and image pattern is complex [7].

In order to deal with complex image patterns, Chan et al. proposed Piecewise Smooth (PS) model [5]. Unfortunately, the PS model based on Euler-Lagrange and gradient descent is so complex that it costs a lot of computation time [8].Li et al. proposed Local Binary Fitting (LBF) model, which uses local information as constrains to deal with inhomogeneous regions

Jun Yang is a postgraduate studying in the Department of Mechanical Engineering, Tsinghua university, China (e-mail:jyang16@mails.tsinghua.edu.cn)

Xuekun $\mathrm{Li}$ is an assistant professor in the Department of Mechanical Engineering and the State Key Lab of Tribology in Tsinghua University. China (corresponding author e-mail: xli@tsinghua.edu.cn).

Zeming Zhao is the general manager of HIECISE Precision Equipment Co. Ltd. China.(e-mail: zeming.zhao@hiecise.com)

Junchuang Chen is a research engineer of HIECISE Precision Equipment Co. Ltd. China.(e-mail: junchuang.chen@hiecise.com)

Yiming Rong is a professor of Mechanical and Energy Engineering in Southern University of Science and Technology. China (e-mail: rongym@su stc.edu.cn) 
[9]. Bresson et al. [7] proposed a similar model to LBF, which combines information on edge and global intensity with an edge indicator function. Zhang et al.[10] proposed the Local Image Fitting (LIF) energy function by minimizing the difference between an original image and the fitted image. In the above mentioned segmentation models, initial curve is always randomly chosen, which may lead to a local optimization rather than a global one. Optical defects on roller surface are usually hard to be detected because of the low contrast between the fine defects and the large high-glaze texture background and between the fuzzy defect boundary and the random spread pattern. Above mentioned models usually cannot work effectively for defect segmentation with low contrasts.

For image classification, the most important task is to build proper eigenvectors, which can describe the image features accurately and achieve a certain degree of differentiation. Gabor filters are proved efficient to defect image processing. Mallat et al. [11] proposed the theoretical breakthrough of wavelet, which has been widely used since the 1990s. Daugman [12] proposed the discrete Gabor wavelet for image processing. Muller et al.[13] raised that Gabor can grasp the spatial frequency and local structure characteristics of multiple directions in the local area of an image. Vetterli et al. [14] raised that amplitude feature is mostly used in image processing because amplitude changes are relatively smooth and steady while space position changes recurrently. However, the extracted eigenvectors are redundant and the dimension is too high to be suitable for operation and training. Jolliffeit et al. [15] proposed the principal component analysis (PCA) method, which is based on the assumption of high information corresponds to high variance $[16,17]$. In PCA, original data are projected to a new coordinate space after PCA feature extraction $[18,19]$. Each coordinate axis in the new coordinate space represents a principal component vector. The first principal component vector contains the maximum variance; the second one is orthogonal to the first $[20,21]$. Therefore, the number of dimensions can be reduced effectively.

BP (back propagation neural network) and SVM (support vector machine) are the popular classification algorithms. However, BP is complex and SVM is slow in training. That limits their applications in real-time monitoring. Lowe et al. [22] proposed RBF (Radial basis function) neural network, which can adaptively adjust connection weights between neurons. However, RBF neural network is slow in learning. Pao. et al. [23] proposed Random Vector Functional Link (RVFL) algorithm, in which training only requires output weight. Huang et al. [24] proposed extreme learning machine (ELM) algorithm. Which are feedforward neural network for classification and feature learning with a single layer or multiple hidden layers, where a parameters of hidden nodes need not be tuned. Huynh et al. [25] pointed out that ELM randomly generates and selects the parameters for the hidden layer according to a certain continuous probability distribution $[-1,1]$. Comparing to SVM and traditional neural networks, ELM requires less manual intervention and has a strong generalization ability for heterogeneous data sets. Furthermore, Huang et al. [26] advanced ELM based on kernel functions.

Comprehending the existing works, we propose an on-site machine vision system for stable inspection of the optical defects on roller surface. The remaining of the paper is organized as follows. Section 2 proposes an improved segmentation and classification model by introducing a penalty term and a convolution kernel with alternative sizes. Section 3 designs an on-site machine vision system to capture the surface images. Section 4 carries out experiments to verify the efficiency of our improved segmentation and classification algorithms. Section 5 concludes the paper.

\section{IMPROVED SEGMENTATION METHOD}

\section{A. Seed contour generation by morphology methods}

Segmentation for roller surface, requires an image segmentation model, which is effective for processing complex pattern, fuzzy boundary and inhomogeneous intensity. Active contour model seems suitable. However, this model randomly sets initial contours so that it may cause wrong results for roller surfaces which contain fuzzy defects. Because the energy function may not converge to the global optimization but the local one. Therefore, we propose a method to generate several "seed contours" as possible defect areas.

Three steps for the morphology pretreatment of an original image are as follows:

- Enhancing the image by "top hat" and "black hat" operations.

- $\quad$ Eliminating salt-and-pepper noise in images with adaptive median filters.

- $\quad$ Eroding and dilating.

Compared with previous random initial contour, seed contour can roughly locate the defects and then evolve towards the boundary of object more precisely.

\section{B. Improved active contour without edges}

Chan-Vese model [5] uses the arithmetic mean values of inside and outside of the current contour as domain intensity i.e., $m_{\text {in }}$ or $m_{\text {out }}$

$$
\begin{aligned}
m_{\text {in }} & =\frac{\int_{\text {Cin }} I(x, y) H(\phi) d x d y}{\int_{\text {Cin }} H(\phi) d x d y} \\
m_{\text {out }} & =\frac{\int_{\text {Cout }} I(x, y)(1-H(\phi)) d x d y}{\int_{\text {Cout }}(1-H(\phi)) d x d y}
\end{aligned}
$$

Formula (1) and (2) only take the global intensity into consideration. That is less effective for image segmentation with complex fuzzy boundaries and inhomogeneous intensity. To achieve effective segmentation, we not only takes local information into consideration by bringing a convolution operation with an alterable kernel size, but also propose a penalty term $\delta$ in order to make every pixel intensity contribution more appropriate.

Penalty term: 


$$
\begin{aligned}
& \delta_{\text {in }}^{i+1}(x, y)=\frac{1}{1+\frac{\left(I(x, y)^{i+1}-m_{\text {in }}^{i}\right)^{2}}{\left(I(x, y)^{i+1}-m_{\text {out }}^{i}\right)^{2}}} \\
& \delta_{\text {out }}^{i+1}(x, y)=\frac{1}{1+\frac{\left(I(x, y)^{i+1}-m_{\text {out }}^{i}\right)^{2}}{\left(I(x, y)^{i+1}-m_{\text {in }}^{i}\right)^{2}}}
\end{aligned}
$$

If $I(x, y)^{i+1}$ is far away from $m_{\text {in }}^{i}$ but close to $m_{\text {out }}^{i}$, $\left(I(x, y)^{i+1}-m_{\text {out }}^{i}\right)^{2}$ will be very small. It leads $\delta_{\text {in }}^{i+1}(x, y)$ to be a small value, so that the contribution of $I(x, y)^{i+1}$ is small when calculating $m_{i n}^{i+1}$. The same happens in calculating $m_{\text {out }}^{i+1}$.

We use a Gaussian convention kernel $\mathrm{K}(u)$ to slide around each pixel to consider its local intensity. Different from the constant kernel size in LBF model, the convention kernel size in our model is adjustable according to the penalty term $\delta(\mathrm{x}, \mathrm{y})$.

$$
\begin{gathered}
K_{\sigma}(u)=\frac{1}{(2 \pi)^{\frac{n}{2}} \sigma^{n}} e^{-|u|^{2} 2 \sigma^{2}} \quad \mathrm{u} \in W_{r \times r} \\
\mathrm{r}=\left\{\begin{array}{cc}
9 & \delta(x, y) \in(0,0.25] \\
7 & \delta(x, y) \in(0.25,0.5] \\
5 & \delta(x, y) \in(0.5,0.75] \\
3 & \delta(x, y) \in(0.75,1)
\end{array}\right.
\end{gathered}
$$

Where $\sigma$ is the Gaussian parameter and $\mathrm{r}$ is the size of convention kernel $(\mathrm{r} \times \mathrm{r}), W_{r \times r}$ is a $\mathrm{r} \times \mathrm{r}$ square window with Gaussian coefficients. The domain intensity is as the following:

$$
\begin{gathered}
m_{\text {in }}{ }^{i+1}=\frac{\int_{\text {Cin }}\left|\delta_{\text {in }}{ }^{i+1}(x, y)\right| K\left(\sigma, \delta_{\text {in }}{ }^{i+1}(x, y)\right) \times I(x, y)^{i+1} H(\phi) d x d y}{\int_{\text {Cin }} H(\phi) d x d y} \\
m_{\text {out }}{ }^{i+1}=\frac{\int_{\text {Cout }}\left|\delta_{\text {out }}{ }^{i+1}(x, y)\right| K\left(\sigma, \delta_{\text {out }}{ }^{i+1}(x, y)\right) I(x, y)^{i+1}(1-H(\phi)) d x d y}{\int_{\text {Cout }}(1-H(\phi)) d x d y}
\end{gathered}
$$

Comparing with the Chan-Vese model, our model makes use of local image information so that it outperforms the ChanVese model when processing images with complex blur boundaries and intensity inhomogeneity.

Using Heaviside function and level set function, the energy function becomes:

$$
\begin{aligned}
& E(x, y, \phi) \\
= & \int|\nabla H(\phi)| \\
+ & \lambda_{1} \int_{\text {in }}\left|I(x, y)-m_{\text {in }}\right|^{2} H(\varnothing) d x d y \\
+ & \lambda_{2} \int_{\text {out }}\left|I(x, y)-m_{\text {out }}\right|^{2}(1-H(\varnothing)) d x d y
\end{aligned}
$$

Where $\mathrm{H}(\mathrm{z})$ and $\partial$ are expressed as follows:

$$
\mathrm{H}(\mathrm{z})=\frac{1}{2}\left(1+\frac{2}{\pi} \arctan \frac{z}{\varepsilon}\right)
$$

$$
\partial=\frac{1}{\pi} \frac{\varepsilon}{\varepsilon^{2}+z^{2}}
$$

Level set function $\phi$ is positive outside a curve and negative inside a curve. It calculates the shortest distance between a pixel position $\mathrm{p}(\mathrm{x}, \mathrm{y})$ and a current curve. It is calculated as follows:

$$
\emptyset(\mathrm{x}, \mathrm{y})=\left\{\begin{array}{cl}
d[(x, y), C] & (x, y) \text { is inside } C \\
-d[(x, y), C] & (x, y) \text { is outside } C
\end{array}\right.
$$

Formula (13) calculates the threshold for stopping the iteration in our algorithm:

$$
\mathrm{P}=\frac{\sum_{\left|\emptyset_{x, y}^{i}\right|<h}\left|\emptyset_{x, y}^{i+1}-\emptyset_{x, y}^{i}\right|}{\operatorname{num}\left\{(x, y)\left|\emptyset_{x, y}^{i}\right|<h\right\}}<h^{2} \tau
$$

Where $\operatorname{num}\left\{(x, y)\left|\emptyset_{x, y}^{i}\right|<h\right\}$ means the number of pixel $(\mathrm{x}, \mathrm{y})$ in the condition of $\left|\emptyset_{x, y}^{i}\right|<h$, indicating that the given curve converges into the boundary of an object. $h^{2} \tau$ is decreasingly set to be 0.1-0.01 on image features.

\section{IMPROVED CLASSIFICATION METHOD}

\section{A. Gabor Filter Feature Vector Extraction}

Gabor filter has its special advantage on image feature extraction and texture analysis. Gabor features include amplitude and power. Amplitude is often used in image feature recognition because amplitude change is relatively smooth and steady while the space position shows a periodic change. Amplitude reflects the image energy spectrum, Gabor amplitude is usually called Gabor energy, which is defined as follows:

$$
\begin{aligned}
\mathrm{g}(x, y, \lambda, \theta, \sigma, \gamma) & =\exp \left(-\frac{x^{\prime 2}+\gamma^{2} y^{\prime 2}}{2 \sigma^{2}}\right) \exp \left(i\left(2 \pi \frac{x^{\prime}}{\lambda}+\psi\right)\right) \\
x^{\prime} & =x \cos \theta+y \sin \theta \\
y^{\prime} & =-x \sin \theta+y \cos \theta
\end{aligned}
$$

$\lambda$ : Sine wave length

$\theta:$ The direction of the Gabor kernel

$\psi:$ phase deviation

$\sigma:$ The standard deviation of the gaussian

\section{$\gamma:$ Space aspect ratio}

Gabor filter is often used in practice. The Gabor function is a wave function actually restricted by Gaussian function. A group of Gabor filters can be obtained by defining different kernel functions.

Gabor kernel function: 


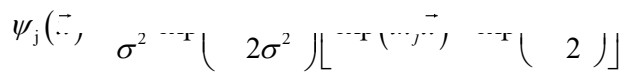

Gabor filter can be defined as:

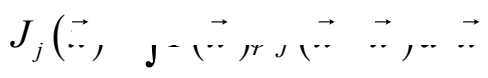

Where:

$\exp \left(-k_{j}^{2} x^{2} /\left(2 \sigma^{2}\right)\right)$ : Gaussian function.

$\therefore$ : location

$I(\vec{\therefore}$, : value of the image field

$\vec{h}_{j}$ : the different response of filters to different scales and directions

A series of Gabor filters can be obtained through the selection of $\vec{h}_{j} \cdot \vec{h}_{j}$ can be defined as follows:

$$
\left.\vec{h}_{j}-k_{j y}\right)=\left(\begin{array}{l}
k_{v} \cos \varphi_{\mu} \\
k_{v} \sin \varphi_{\mu}
\end{array}\right)
$$

$v$ controls the wavelength of a window, so it controls sampling length (frequency). $\mu$ controls sampling direction by controlling the oscillation direction of an oscillating function. The Gabor filter uses the convolution formula to obtain different filtering results for each image position.

\section{B. Kernel Principal Component Analysis}

KPCA feature extraction will take the following four steps:

- Map the original sample to a linearly separable high dimensional space

$$
\mathrm{X}=\left[\Phi(X), \Phi\left(X_{2}\right) \ldots \Phi\left(X_{m}\right)\right]
$$

Where $\Phi(\mathrm{x})$ is the selected kernel function.

- Use the positive nucleation K.to calculate the eigenvalues of matrix $\mathrm{R}$ and select the eigenvalues after ranking, then calculate its corresponding eigenvector.

$$
R=K-K \cdot M-M \cdot K+M \cdot K \cdot M
$$

- Compute the eigenvalues and eigenvectors of covariance matrix $\mathrm{C}$ based on formula (22)

$$
w_{i}=\lambda_{i}^{-1 / 2} Q v_{i}(i=1,2, \cdots, \mathrm{d})
$$

where $\mathrm{C}=m^{-1} Q Q^{T}$ is the covariance matrix. $W=\left[w_{1}, w_{2}, \cdots, w_{p}\right]$ is the feature extraction matrix used in feature extraction algorithm.

- $\quad$ For $\forall x_{i} \in X, \mathrm{i}=1,2, \cdots, \mathrm{m}$, calculate $y_{1}, y_{2}, \cdots, y_{m}$ according to $y_{i}=W^{T} \Phi\left(x_{i}\right), \quad Y=\left[y_{1}, y_{2}, \cdots, y_{m}\right]$ is the output feature set, the algorithm end.

\section{ELM neural networks}

Assume there are $\mathrm{N}$ arbitrary samples $\left(x_{i}, t_{i}\right)$.Among them, $X_{i}=\left[x_{i 1}, x_{i 2}, \ldots, x_{i n}\right]^{T} \in R^{n}, t_{i}=\left[t_{i 1}, t_{i 2} \ldots, t_{i m}\right]^{T} \in R^{m} . \mathrm{A}$ SLFN (Single-hidden Layer Feed forward Neural Networks) with L nodes on the hidden layer (Fig.1) can be expressed as formula (23)

$$
\sum_{i=1}^{L} \beta_{i} g\left(W_{i} \cdot X_{j}+b_{i}\right)=o_{j}, j=1, \ldots, N
$$

Where:

$\mathrm{g}(\mathrm{x})$ : activaion function

$W_{i}=\left[w_{i, 1}, w_{i, 2}, \ldots, w_{i, n}\right]^{T}:$ Input weights

$\beta_{i}$ :Output weights

$b_{i}$ :Bias of the i-th implicit layer unit

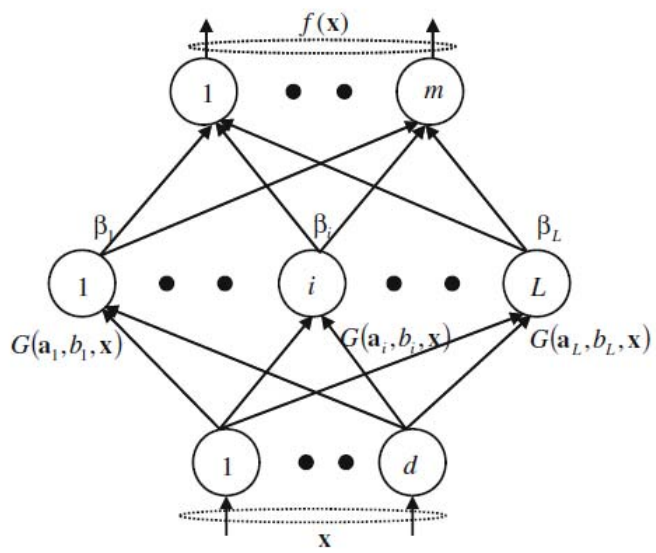

Figure 1 . the schematic diagram of the ELM algorithm

The training process is as follows:

a. Input: Training sample set $\left\{\left(x_{i}, t_{i}\right)\right\}_{i=1}^{N} \subset R^{n} \times R^{m}$, hidden layer output function $\mathrm{G}(\mathrm{a}, \mathrm{b}, \mathrm{x})$ and number of nodes in the hidden layer.

b. Generate parameters $\left(a_{i}, b_{i}\right)$ randomly for hidden layer nodes, $\mathrm{i}=1,2 \ldots \mathrm{L}$

c. Calculate hidden layer output matrix $\mathrm{H}($ ensure $\mathrm{H}$ is with full rank)

d. Output: Network optimal power $\beta$ : $\beta=H^{T} T$

Although ELM originates from the BP neural network, but it randomly generates parameters for the hidden layer nodes, which greatly simplifies the complex iterative process in traditional neural network.It can satisfy the double requirements of accuracy rate and calculation speed.

\section{EXPERIMENT SETUP AND RESULTS ANALYSIS}

Fig. 2 illustrates an on-site machine vision inspection system, which contains a linear blue light source, a line-scan camera, an encoder and a computer. The parameters of the light source and specifications of the line-scan camera are shown in Fig. 3.

The imaging process is as follows: the monochromatic light source emits linear blue light, which projects on the 
grinding roller surface along the axial direction. The reflected light will be received by the line-scan camera, which has only one row of CMOS photosensitive cell and gets pixel rows as the roller rotates simultaneously. Then the camera splices the pixel rows into an entire image and transmit the image to the computer for further processing.

We carried out experiments several times for the roller surface defect inspection. Finally we chose $30 \mathrm{kHz}$ as the data acquisition frequency and 40rpm as the roller rotation speed. The algorithms run on the MATLAB R2017a. The program runs on a ThinkPad P50s with Core i7@2.5GHz CPU and 16GB memory.

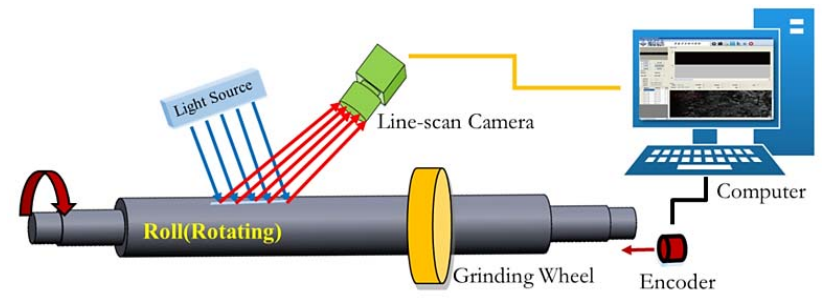

Figure 2 Real-time machine vision-based roller surface monitoring system

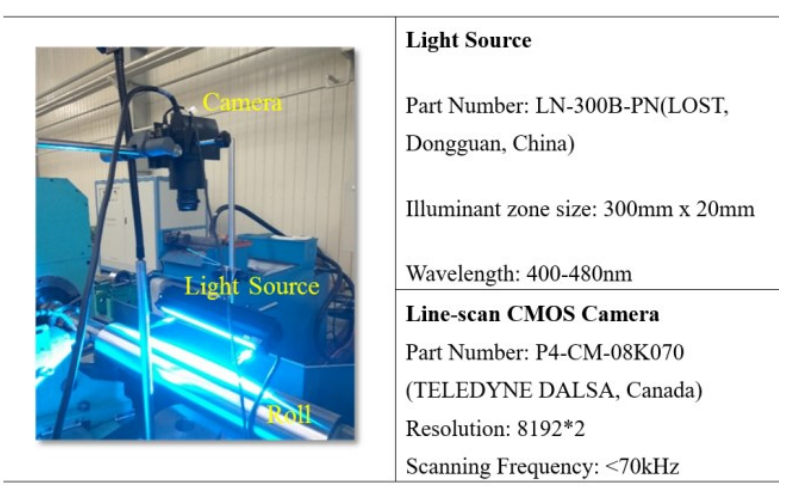

Figure 3 Experiment setting

The part images with defects are also shown as Fig. 4(a), (b) and (c). Note that the images in Fig. 4 are cropped in different scale for better visualization. ((a): $600 \times 600$; (b): $1200 \times 1200$; (c): $1800 \times 1800$ )

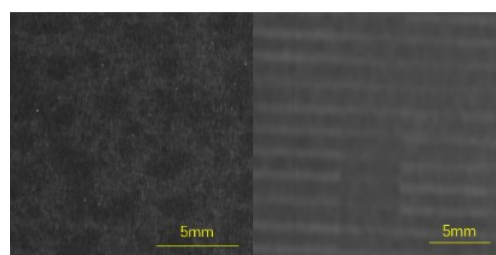

(a) (b)

Figure 4 Images defects

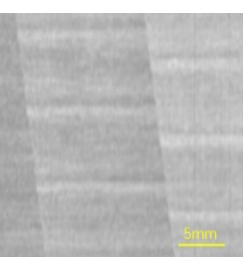

(c) (a) speckles (b)chatters (c) combination of chatters and feed traces.

As illustrated in Fig. 4, the optical defects on roller surface has low intensity contrast, with fuzzy blur boundary and random or regular complex pattern, which is hard to segment directly. Therefore, before executing segmentation, we preprocess original images to augment defect features. Fig. 5 presents the preprocessed results for the above three defects.

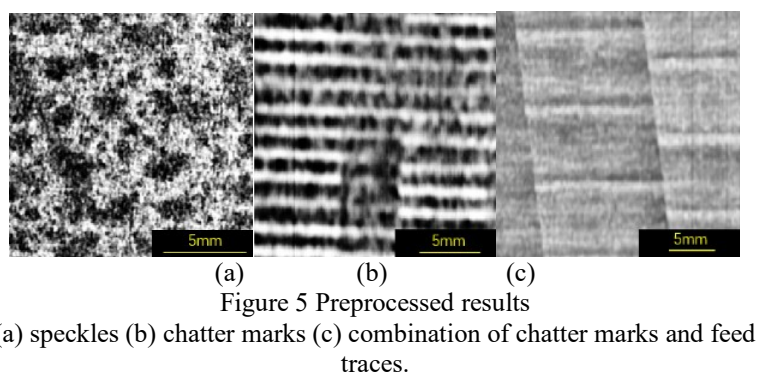

Fig. 6 presents the results with our improved segmentation model:

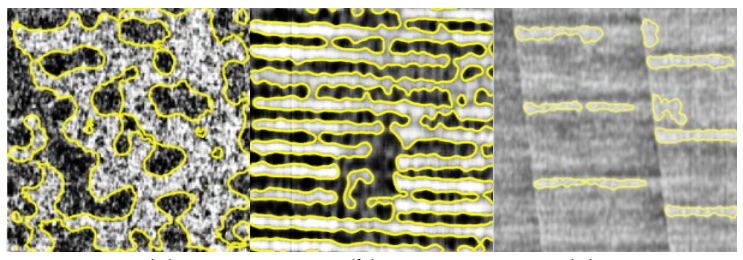

(a)

(b)

(c)

Figure 6 Segmentation results with our improved model

(a) speckles (b) chatters (c) combination of chatter and feed traces.

TABLE 1. ITERATION STEPS AND TIME.

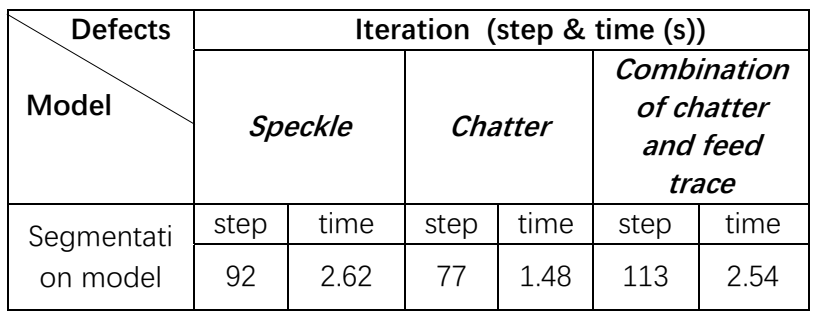

Segmentation results in Fig. 6 show that our segmentation method can effectively get correct results. Tablel shows the iteration steps and consuming time. We set the same iteration stop condition for different defects. The energy variation is less than a constant threshold, $h^{2} \tau=0.016$. Our method can converge under global optimization with an acceptable time.

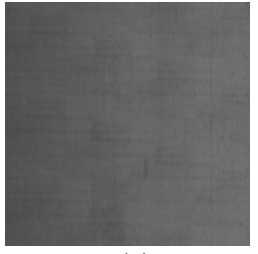

(a)

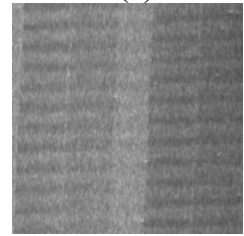

(c)

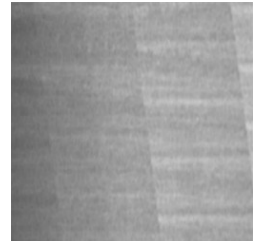

(b)

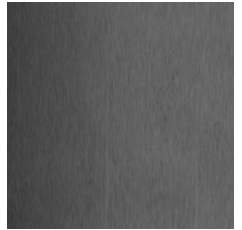

(d)
Figure 7 Image samples with different defects (a)Cross grains (b) swirl mark (c) chatters (d) normal image

The above mentioned algorithm requires to operate on square images. However, the amount of sample images cannot meet the condition, Meanwhile, partial original image area do not contain defects or is under exposed on the edge. So we 
capture 100 images with each defects and non-defective images (the pixel resolution is $256 * 256$ ), for validation testing. Table 2 present the parameters and recognition rates.

TABLE 2 RECOGNITION RATES UNDER DIFFERENT CONDITIONS

\begin{tabular}{|c|c|c|c|c|}
\hline Parameter & kernel & repetition & $\begin{array}{c}\text { Verified } \\
\text { image }\end{array}$ & $\begin{array}{c}\text { recognitio } \\
\text { n rate }\end{array}$ \\
\hline 1 & Gaussian & 50 & 1 & $82.92 \%$ \\
\hline 2 & Gaussian & 50 & 2 & $81.26 \%$ \\
\hline 3 & Gaussian & 100 & 1 & $84.53 \%$ \\
\hline 4 & Gaussian & 100 & 2 & $82.67 \%$ \\
\hline 5 & $\begin{array}{c}\text { polynomia } \\
1\end{array}$ & 50 & 1 & $74.50 \%$ \\
\hline 6 & $\begin{array}{c}\text { polynomia } \\
1\end{array}$ & 50 & 2 & $72.75 \%$ \\
\hline 7 & $\begin{array}{c}\text { polynomia } \\
1\end{array}$ & 100 & 1 & $77.45 \%$ \\
\hline 8 & $\begin{array}{c}\text { polynomia } \\
1\end{array}$ & 100 & 2 & $76.83 \%$ \\
\hline
\end{tabular}

Line chart of recognition rates

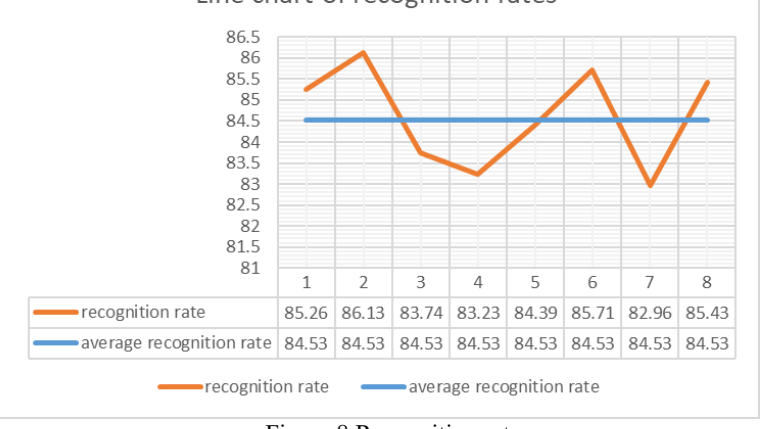

Figure 8 Recognition rates

As shown in Table 2, the para-meters contain kernel, repetition times and the number of verified image. The kernel in the experiment are Gaussian and polynomial. The repetition is determined by the recognition times and the sample number. The number of images represents the number of validations in each group. The results show that with the Gaussian kernel, 100 repetitions and 1 verified image, the recognition can reach the highest recognition rate (Fig. 8).

In the experiment, the recognition rate is about $80 \%$, which is not very good. The reason might be as follows: the adopted Gabor filters mainly extract texture features. Although chatter and swirl marks are similar to texture, they may cause misclassification.

\section{CONCLUSION}

The surface quality of steel roller is significant for finish products. This paper proposes an improved method for monitoring grinding roller surface. First, we build an on-site machine vision-based roller surface inspection platform for capturing roller surface images. Second, we propose an improved defect segmentation model, which takes the penalty term and the convolution kernel into consideration. In defect classification, we adopt the Gabor wavelet method with KPCA algorithm to extract image features, which reduces redundant eigenvectors greatly. In the experiment, we test our defect segmentation method for three typical defects: speckles, chatter marks and the combination of chatter marks and feed traces. Each type of defect has fuzzy boundary, complex pattern and intensity homogeneity, which are hard to segment. Results verify that the energy function proposed in our model can converge under global optimization instead of local one, with an acceptable inspection time. In the defect classification of the images with cross grain, chatter marks and swirl marks, we adopt three parameters - kernel, repetition and the number of verified image. The results show that the classification under Gaussian kernel, 100 repetitions and 1 verified image can reach the highest rate at $84.53 \%$.

\section{ACKNOWLEDGMENT}

This research is supported by Project NSAF-U1430116 and Project 2017ZX04007001. The authors would also like to thank the support from Tsinghua University Initiative Scientific Research Program and Tsinghua-RWTH Aachen Collaborative Innovation and Southern University of Science and Technology Funding.

\section{REFERENCES}

[1] M. Kass, A. Witkin and D. Terzopoulos, "Snakes: Active Contour Models," Inter. J. Comp. Vi. vol.1,2001, pp.321-332.

[2] R. Malladi, J.A. Sethian and B.C. Vemuri, "A topology independent shape modeling scheme. " Proc. SPIE Conf. Geo. Methods Comp. Vi. II, vol. 2031,1993, pp. 246-258

[3] Y. Shi and W.C. Karl, "A Fast Level Set Method Without Solving PDEs," Proceedings. (ICASSP '05). IEEE International Conference on Acoustics, Speech, and Signal Processing, 2005, pp. 97-100.

[4] V. Caselles, R. Kimmel and G. Sapiro, "Geodesic active contours," Proceedings of IEEE International Conference on Computer Vision, Cambridge, MA, 1995, pp. 694-699.

[5] T. F. Chan and L. A. Vese, "Active contours without edges,"IEEE Transactions on Image Processing, vol. 10, no. 2,2001, pp. 266-277.

[6] T. F. Chan and L. A. Vese, "A level set algorithm for minimizing the Mumford-Shah functional in image processing," Proceedings IEEE Workshop on Variational and Level Set Methods in Computer Vision, Vancouver, BC, 2001, pp. 161-168.

[7] X. Besson, S. Esedoglu and P. Vandergheynst,"Fast Global Minimization of The Active Contour/Snake Model, "J. Math. Ima. Vi. vol. 28,2007, pp. 151-167.

[8] T. Chan, S. Esedoglu and M. Nikolova, "Algorithms for Finding Global Minimizers of Image Segmentation and Denoising Models, " J. App. Math. vol. 66, 2006, pp. 1632-1648.

[9] C. Li, C. Kao and J. Gore, "Implicit Active Driven by Local Binary Fitting Energy. "Proc. IEEE Conf. Comp. Vi. and Pat. Recog, 2007, pp. $1-7$

[10] K. Zhang, H. Song, L. Zhang, "Active contours driven by local image fitting energy. " Pat. Recog., 2010, pp. 1199-1206 .

[11] S. Mallat. "A theory for multiresolution signal decomposition : the wavelet representation, " IEEE Transactions on Pattern Analysis and Machine Intelligence, vol. 11,1989, pp. 674-693.

[12] J.G.Daugman. "Complet Discrete 2-D Gabor Transforms by Neural Networks for image analysis and compression," IEEE Trans on ASSP, vol. 36, 1988, pp. 1169-1179.

[13] H. Muller, N. Milhous and D. Bandon, "A review of content-based image retrieval systems in medical applications. clinical benefits and future directions, " International Journal of Medical Information, vol. 73,2004 , pp. $1-23$ 
[14] M. Vetterli and J. Kovacevic, "Wavelets and Subband Coding, " Englewood Cliffs, NJ, Prentice-Hall,1995, pp. 86-124

[15] I. Jolliffeit. "Principal Component Analysis" New York: Springer, 1986, pp.14-32

[16] R Manthalker, P.K. Biswas, B.N. Chatterji, " Rotation invariant texture classification using even symmetric Gabor filters, "Pattern Recognition Letters, vol. 24, 2003, pp. 2061-2068.

[17] H. Hotelling, "Analysis of a complex of statistical variables into principal components. " J. Educ. Psychol, vol. 24, no. 6, pp. 417-441, 1993.

[18] I. T. Jolliffe, "Principal Component Analysis, " New York, SpringerVerlag,2002.

[19] A. K. Mishra and B. Mulgrew, "Radar signal classification using PCAbased features," proceeding of ICASSP, IEEE International Conference on Acoustics, Speech and Signal Processing, 2006, pp.1104-1107.

[20] C.Z. Qiu, H. Ren and H. Zou, "Performance Comparison of Target Classification in SAR Images Based on PCA and 2D-PCA Features," IEEE Asian-pacific Conference on Synthetic Aperture Radar, 2010, pp. 868-871.

[21] A. Aspremont, F. Bach, and L. E. Ghaoui. "Full regularization path for sparse principal component analysis." Proc. Int. Conf. Mach. Learn.2007, pp. 177-184.

[22] D. Lowe, "Adaptive radial basis function nonlinearities, and the problem of generalisation," 1989 First IEE International Conference on Artificial Neural Networks, (Conf. Publ. No. 313), London, 1989, pp. 171-175.

[23] Y.H. Pao, G.H. Park and D.J. Sobajic, "Learning and generalization characteristics of the random vector functional-link net," Neurocomputing,vol. 6, 1994, pp. 163-180.

[24] G.B.Huang, Q.Y. Zhu and C.K. Siew, " Extreme learning machine: a new learning scheme of feedforward neural networks. " IEEE International Joint Conference on Neural Networks, 2004, pp. 985-990

[25] H.T. Huynh, Y. Won, J.J. Kim, "An improvement of extreme learning machine for compact single-hidden-layer feed forward neural networks. " International journal of neural systems, vol. 18, no. 5, 2008,pp. 433441.

[26] G.B. Huang, Q.Y. Zhu, C.K. Siew, "Extreme learning machine: Theory and applications. " Neurocomputing, vol. 70, 2006, pp. 489-501.

[27] R. Kohavi, " A study of cross-validation and bootstrap for accuracy estimation and model selection, "International Joint Conference on Artificial Intelligence, vol. 14,1995, pp. 1137-1143. 